\title{
A Case of $\boldsymbol{H}$. pylori-associated Granulomatous Gastritis with Hypertrophic Gastropathy
}

\author{
Yeon Soo Kim*, Hye Kyung Lee ${ }^{\dagger}$, Jong $\mathrm{Ok} \mathrm{Kim}^{\dagger}$, Seung Woo Lee*, Sang Beom Kang*, Soon Woo \\ Nam*, and Dong Soo Lee* \\ Departments of *Internal Medicine and ${ }^{\dagger}$ Pathology, Daejeon St. Mary's Hospital, College of Medicine, The Catholic University of Korea, \\ Daejeon, Korea
}

A 46-year-old man had chronic granulomatous gastritis characterized by giant gastric folds with noncaseating epithelioid granulomas including giant cells in the corpus. No definite etiologic factors were detected. Histology and the rapid urease test indicated that $H$. pylori was present in both the antrum and corpus. The granulomatous gastritis with giant gastric folds improved after $H$. pylori eradication. This case suggests an association between isolated granulomatous gastritis and H. pylori infection. (Gut and Liver 2009;3:137-140)

Key Words: Granulomatous gastritis; Helicobacter pylori; Hypertrophic gastritis

\section{INTRODUCTION}

Chronic granulomatous gastritis (CGG) is a rare disease. $^{1,2}$ It constitutes about $0.3 \%$ of all cases of gastritis and is characterized by the presence of granulomas. ${ }^{2,3}$ Idiopathic granulomatous gastritis (IGG) refers to a chronic granulomatous reaction limited to the stomach without the identification of specific underlying causes, ${ }^{4}$ therefore its pathogenesis and the optimal treatment is controversial. Currently, Helicobacter pylori (H. pylori) infection is considered a cause of chronic granulomatous gastritis and the associated giant gastric folds. ${ }^{5-7}$ Here, we report a case of CGG that improved after $H$. pylori eradication.

\section{CASE REPORT}

A 46-year-old man was transferred to the department of Internal Medicine with the suspicion of gastric cancer. By the routine Upper gastrointestinal series, an ill-defined mass like lesion in the gastric corpus was detected. There was no specific past history and the patient did not complain of any significant symptoms. Physical examination and laboratory findings were within normal limits. The upper endoscopy showed an ill-defined $4 \times 3 \mathrm{~cm}$ area with regional hypertrophied gastric folds at the great curvature from the lower to the mid body of the stomach (Fig. 1A). The mass had a soft consistency noted with the endoscope. The upper gastrointestinal series and abdominal CT showed mucosal fold thickening at the great curvature and lower body of the stomach (Fig. 2). The gastric biopsy tissue obtained showed several granulomas in five biopsy fragments. The diameter of the granulomas was variable. The smallest measured less than $0.10 \mathrm{~mm}$ in diameter. The range of sizes was $0.10-0.40 \mathrm{~mm}$. The granulomas were localized either superficially, i.e., just underneath the surface epithelium, or deeply, down to the level of the foveolar isthmi. They consisted of a diffuse cellular infiltrate, a circumscribed aggregate or a compact 'sarcoid-like' aggregate (Fig. 3A). The results of the PCR study for Mycobacterium tuberculosis were negative. Other study (colonoscopy, small bowel series) shows no abnormal finding. However, $H$. pylori testing were positive both in the antrum and in the corpus by histology and rapid urease test (Fig. 3B). The patient was treated with triple therapy (rabeprazole $20 \mathrm{mg}+$ amoxicillin 1,500 mg + clarithromycin $600 \mathrm{mg}$ for 1 week) for H. pylori eradication. Follow-up upper endoscopy, after two months, showed decreased gastric fold thickening and the gastric atrophy was improved. However, the biopsy specimens showed focal granulomas without $H$. pylori detected. The endoscopy findings and granulomas all improved one year

\footnotetext{
Correspondence to: Hye Kyung Lee

Department of Pathology, Medical College of Eulji University, 1306, Dunsan-dong, Seo-gu, Daejeon 302-799, Korea

Tel: +82-42-611-3460, Fax: +82-42-611-3459, E-mail: apw01@hanmail.net
}

Received on February 4, 2008. Accepted on March 16, 2009. 

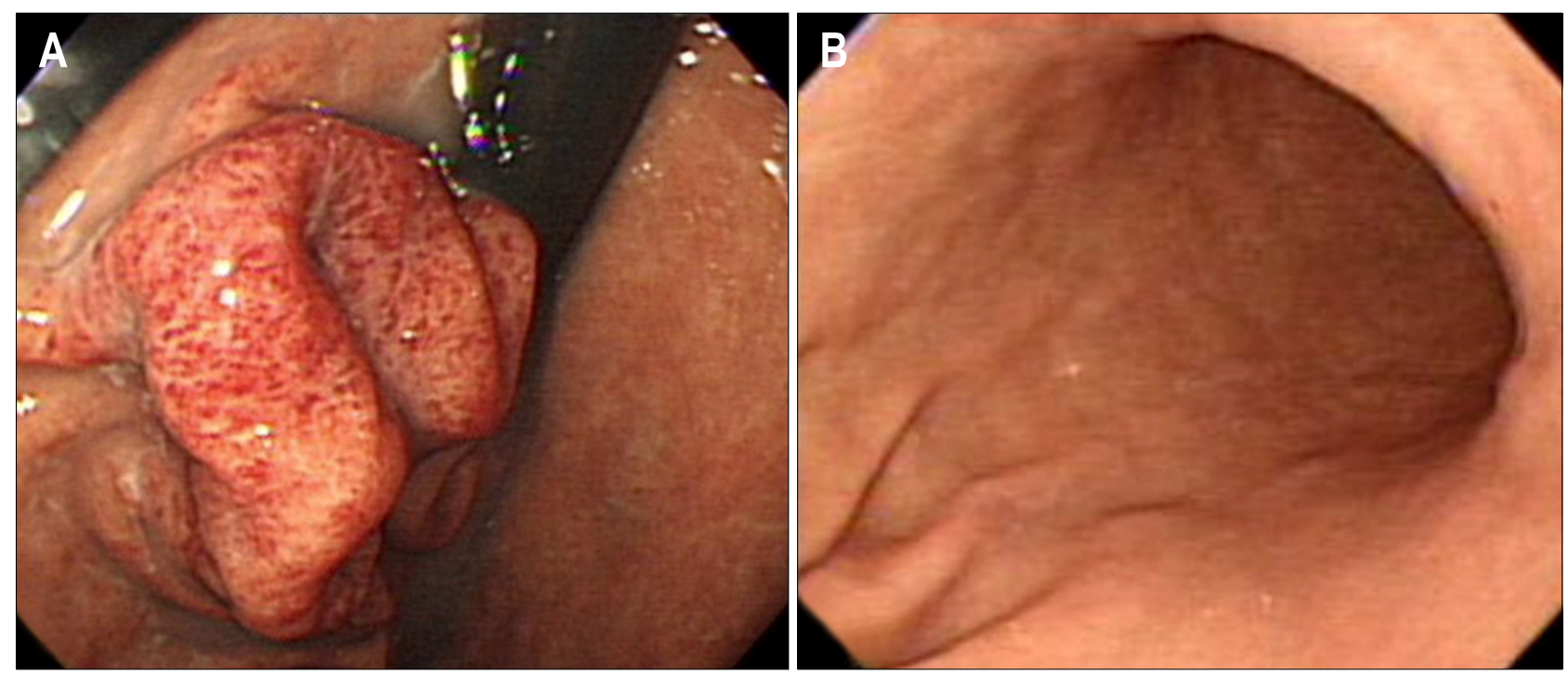

Fig. 1. (A) Upper gastrointestinal endoscopy showing focal enlarged gastric rugae with hyperemic changes at the greater curvature side of the lower body of the stomach. (B) Upper gastrointestinal endoscopy showing that the giant fold improved after $H$. pylori eradication.
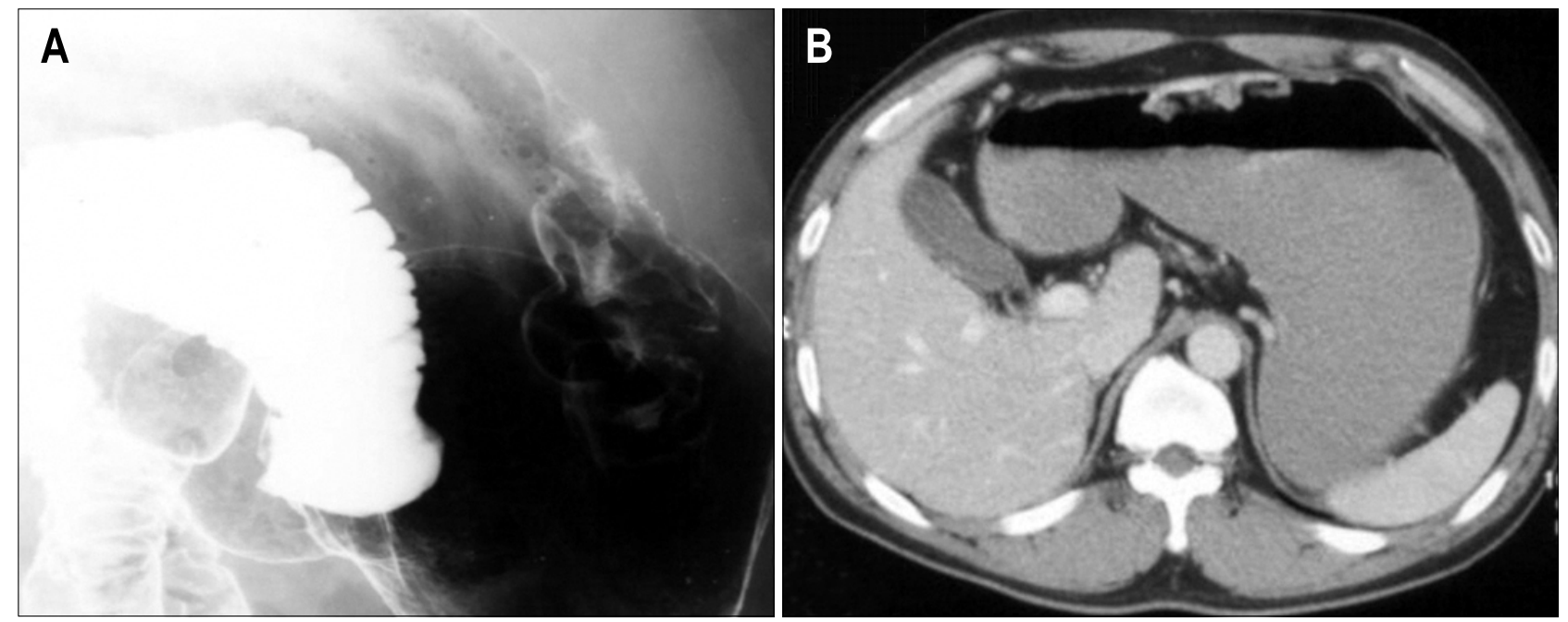

Fig. 2. (A) Double-contrast barium study showing focal thickened and irregular folds at the greater curvature side of the lower body of the stomach. (B) Abdomen tomography showing thickened folds at the greater curvature side of the lower body.

later after H. pylori eradication therapy (Fig. 4).

\section{DISCUSSION}

Chronic granulomatous gastritis (CGG) is uncommon. It may occur in association with several disorders including Crohn's disease, sarcoidosis, infectious diseases, foreign body reaction, malignancy and vasculitis. In the West, the common causes of chronic granulomatous gastritis are Crohn's disease and sarcoidosis, ${ }^{2}$ but in the East, the incidence of $H$. pylori infection has been reported to be high with CGG without the presence of sys- temic illness. ${ }^{7}$ our case was negative for immunohitochemical staining (CD20, CD21, CD35) and had no other underlying disorder (Crohn's disease) for upper gastrointestinal series and colonoscopy.

The association between CGG and $H$. pylori was first reported in $1989 .{ }^{5}$ A high percentage of $H$. pylori organism was identified in all-chronic granulomatous gastritis cases whatever the diagnosis was. H. pylori was detected in $90 \%$ and chronic gastritis with atrophy was present in $95 \%$ of biopsy specimens. ${ }^{3}$ Miyamoto et al. ${ }^{6}$ reported two cases of IGG that completely resolved after $H$. pylori eradication.

The pathogenesis of granuloma formation with $H$. pylori 

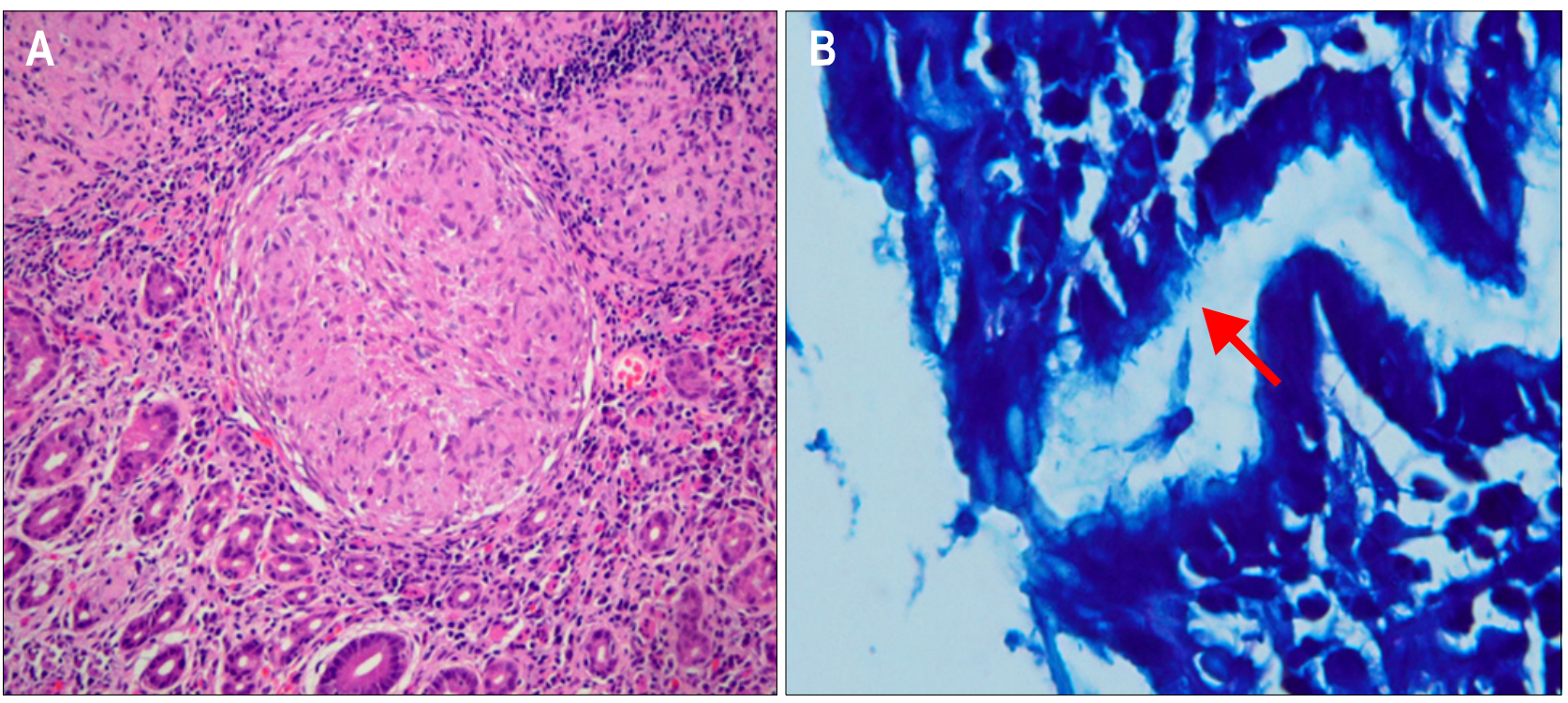

Fig. 3. (A) Endoscopy biopsy showing granulomas consisting of a diffuse cellular infiltrate, a circumscribed aggregate, or a compact 'sarcoid-like' aggregate (H\&E stain, $\times 200)$. (B) H. pylori was evident at the gastric mucosa (Gimsa stain, $\times 400)$.
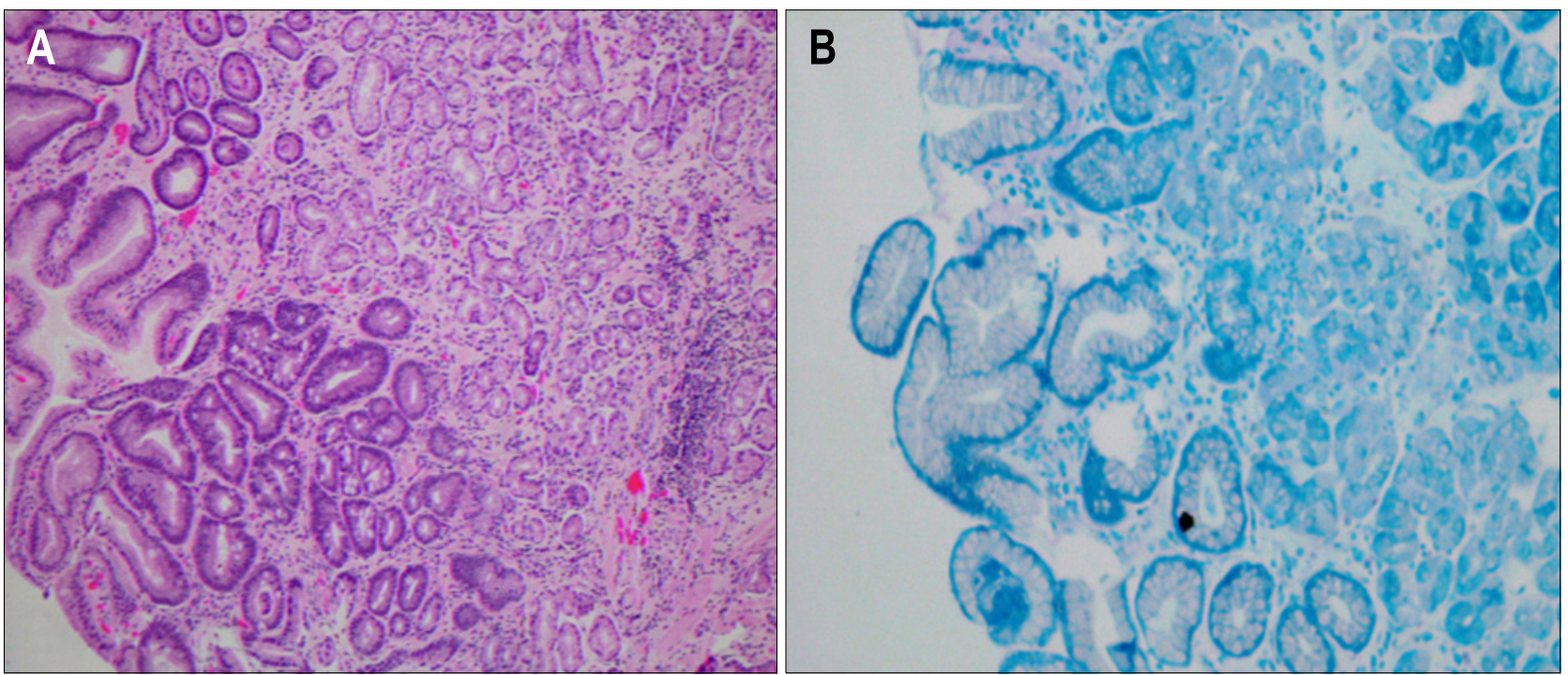

Fig. 4. (A) Endoscopy biopsy showing that the granulomas all improved with mucosal atrophy (H\&E stain, $\times 100)$. (B) $H$. pylori had disappeared from the gastric mucosa (Gimsa stain, $\times 200)$.

infection has not been explained. Granulomas are thought to result from sustained tissue irritation from poorly degradable substances. ${ }^{7}$ The $H$. pylori organism appears to be parasite with flagella and, gastric mucosa injury possibly caused by flagella motility due to persistent immune stimulation from the chronic granulomatous inflammation.

However, the granulomatous lesions persisted for at least 17 months or more after $H$. pylori eradication therapy in our case. In addition, it has been shown that the incidence of $H$. pylori in the population is very high while the frequency of cases of granulomatous gastritis in the population of patients with $H$. pylori-associated gastritis is very low or uncommon. ${ }^{8}$

The cases of CGG gastritis have been equal in both genders and were evenly distributed from the ages of 24 to $69 .{ }^{9}$ In the majority of cases, endoscopic finding are shallow ulcerations, flat erosions, erythemas, but giant gastric folds are rarely appearance like in our case. ${ }^{7}$

Giant gastric folds were first described by Menetrier as an endoscopic finding in patients with hypertrophic gastritis. ${ }^{10}$ They have been observed in other conditions 
including gastrinoma, gastric lymphoma, histoplasmosis, secondary syphilis, anisakiasis, Borrmann type IV, scirrhous carcinoma, and granulomatous gastritis (menetrier disease, hypertrophic gastritis). ${ }^{11}$ In particular, giant fold formation in the fundus and corpus of the stomach might be a consequence of severe high-grade active $H$. pylori gastritis; that is, rare atypical reaction to the colonization of the gastric mucosa with $H$. pylori. ${ }^{12}$ Eradication of the $H$. pylori infection in such patients should result not only in an improvement in the findings associated with gastritis, but also in regression of the giant folds. ${ }^{13}$ Therefore, $H$. pylori infection appeared to be a more important cause of CGG, and idiopathic granulomatous gastritis may be another manifestation of $H$. pylori gastritis. The case reported here is the first report of CGG with giant folds that improved after $H$. pylori eradication.

This case illustrated that CGG appears to be a special form of $H$. pylori gastritis. If underlying diseases was not founded for whole work-up, we firstly consider H. pylori eradication for patients with CGG and H. pylori infection.

\section{REFERENCES}

1. Fahimi HD, Deren JJ, Gottlieb LS, Zamcheck N. Isolated granulomatous gastritis: its relationship to disseminated sarcoidosis and regional enteritis. Gastroenterology 1963; 45:161-175.

2. Shapiro JL, Goldblum JR, Petras RE. A clinicopathologic study of 42 patients with granulomatous gastritis: is there really 'idiopathic' granulomatous gastritis? Am J Surg Pathol 1996;20:462-470.
3. Ectors NL, Dixon MF, Geboes KJ, Rutgeerts PJ, Desmet VJ, Vantrappen GR. Granulomatous gastritis: a morphological and diagnostic approach. Histopathology 1993;23: 55-61.

4. Hirsch BZ, Whitington PF, Kirschner BS, Black DD, Bostwick DG, Yousefzadeh DK. Isolated granulomatous gastritis in an adolescent. Dig Dis Sci 1989;34:292-296.

5. Dhillon AP, Sawyerr A. Granulomatous gastritis associated with Campylobacter pylori. APMIS 1989;97:723-727.

6. Miyamoto M, Haruma K, Yoshihara M, et al. Isolated granulomatous gastritis successfully treated by Helicobacter pylori eradication: a possible association between granulomatous gastritis and Helicobacter pylori. J Gastroenterol 2003;38:371375.

7. Maeng L, Lee A, Choi K, Kang CS, Kim KM. Granulomatous gastritis: a clinicopathologic analysis of 18 biopsy cases. Am J Surg Pathol 2004;28:941-945.

8. Koyama S, Nagashima F. Idiopathic granulomatous gastritis with multiple aphthoid ulcers. Intern Med 2003;42: 691-695.

9. Gumaste VV, Janowitz HD, Waye JD. Granulomatous gastritis: a case report and review of the literature. Am J Gastroenterol 1989;84:1315-1318.

10. Menetrier P. Des polyadenomes gastriques et leurs rapports avec le cancer de stomach. Arch Physiol Norm Path 1888;1:32-35, 236-262.

11. Songür $\mathrm{Y}$, Okai $\mathrm{T}$, Watanabe $\mathrm{H}$, Motoo $\mathrm{Y}$, Sawabu $\mathrm{N}$. Endosonographic evaluation of giant gastric folds. Gastrointest Endosc 1995;41:468-474.

12. Stolte M, Bätz C, Eidt S. Giant fold gastritis: a special form of Helicobacter pylori associated gastritis. Z Gastroenterol 1993;31:289-293.

13. Stolte M, Bätz CH, Bayerdörffer E, Eidt S. Helicobacter pylori eradication in the treatment and differential diagnosis of giant folds in the corpus and fundus of the stomach. $\mathrm{Z}$ Gastroenterol 1995;33:198-201. 